\title{
BF gravity with Immirzi parameter and cosmological constant
}

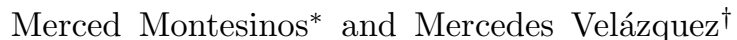 \\ Departamento de Física, Cinvestav, Instituto Politécnico Nacional 2508, \\ San Pedro Zacatenco, 07360, Gustavo A. Madero, Ciudad de México, México.
}

(Dated: November 1, 2018)

\begin{abstract}
The action principle of the BF type introduced by Capovilla, Montesinos, Prieto, and Rojas (CMPR) which describes general relativity with Immirzi parameter is modified in order to allow the inclusion of the cosmological constant. The resulting action principle is on the same footing as the original Plebanski action in the sense that the equations of motion coming from the new action principle are equivalent to the Holst action principle plus a cosmological constant without the need of imposing additional restrictions on the fields. We consider this result a relevant step towards the coupling of matter fields to gravity in the framework of the CMPR action principle.
\end{abstract}

PACS numbers: 04.60.-m, 04.60.Pp

The research in quantum gravity led by its two main branches (loop quantum gravity [1] and spin foam models for gravity [2]) has recently motivated the study of the classical descriptions for general relativity and theories related to it, particularly, the formulations of gravity of the BF type. For instance, Cartan's equations in the framework of BF theories are analyzed in Refs. 3, 4] while the relationship of general relativity to the Husain-Kuchar model in the framework of BF theory is analyzed in Refs. [5 7].

As is well know, general relativity was formulated as a constrained BF theory by Plebański in the mid-seventies [8]. The fundamental variables used for the description of the gravitational field are two-form fields, a connection one-form, and some Lagrange multipliers. This framework was extended much later in order to include the coupling of matter fields [9]. These formulations are complex and one has to use reality conditions in order to get real general relativity. There are other formulations for gravity expressed as a constrained BF theory which are real, and were given in Refs. 10, 11 and 12. In particular, the one given in Ref. 12 includes the so-called Immirzi parameter [13 15]. Recently, the issue of the introduction of the cosmological constant in the framework of Ref. 12 was studied by Smolin and Speziale [16]. This issue is also the goal of this paper. Our approach is different from the one followed in Ref. 16 and, in our opinion, is close to the original Plebański formulation. So, we are ready to make the introduction of the cosmological constant and, at the end of the paper, we will comment on the relationship between the results of this paper and the ones of Ref. 16.

The action principle for pure gravity introduced by Capovilla, Montesinos, Prieto, and Rojas in Ref. 12 (hereafter CMPR) is given by

$$
S[Q, A, \psi, \mu]=\int_{\mathscr{M}^{4}}\left[Q^{I J} \wedge F_{I J}[A]-\frac{1}{2} \psi_{I J K L} Q^{I J} \wedge Q^{K L}-\mu\left(a_{1} \psi_{I J}^{I J}+a_{2} \psi_{I J K L} \varepsilon^{I J K L}\right)\right],
$$

where $A^{I}{ }_{J}$ is a Euclidean or Lorentz connection one-form, depending on whether $S O(4)$ or $S O(3,1)$ is taken as the internal gauge group, and $F^{I}{ }_{J}[A]=d A^{I}{ }_{J}+A^{I}{ }_{K} \wedge A^{K}{ }_{J}$ is its curvature; the $Q$ 's are a set of six two-forms on account of their antisymmetry $Q^{I J}=-Q^{J I}$; the Lagrange multiplier $\psi_{I J K L}$ has 21 independent components due to the properties $\psi_{I J K L}=\psi_{K L I J}, \psi_{I J K L}=-\psi_{J I K L}$, and $\psi_{I J K L}=-\psi_{I J L K}$; the Lagrange multiplier $\mu$ implies the additional restriction $a_{1} \psi_{I J}{ }^{I J}+a_{2} \psi_{I J K L} \varepsilon^{I J K L}=0$ on the Lagrange multiplier $\psi_{I J K L}$. The Lorentz (Euclidean) indices $I, J, K \ldots$ are raised and lowered with the Minkowski (Euclidean) metric $\left(\eta_{I J}\right)=\operatorname{diag}(\sigma,+1,+1,+1)$, where $\sigma=+1$ for Euclidean and $\sigma=-1$ for Lorentzian signatures.

The variation of the action (11) with respect to the independent fields gives the equations of motion

$$
\begin{aligned}
& \delta Q: F_{I J}[A]-\psi_{I J K L} Q^{K L}=0, \\
& \delta A: D Q^{I J}=0, \\
& \delta \psi: Q^{I J} \wedge Q^{K L}+2 a_{1} \mu \eta^{[I|K|} \eta^{J] L}+2 a_{2} \mu \varepsilon^{I J K L}=0, \\
& \delta \mu: a_{1} \psi_{I J} I J+a_{2} \psi_{I J K L} \varepsilon^{I J K L}=0 .
\end{aligned}
$$

It was shown in Ref. 12 that the solution of Eq. (4) for the two-forms $Q^{I J}$ is given by

$$
Q^{I J}=\alpha^{*}\left(e^{I} \wedge e^{J}\right)+\beta e^{I} \wedge e^{J},
$$

\footnotetext{
*Electronic address: merced@fis.cinvestav.mx

${ }^{\dagger}$ Electronic address: mquesada@fis.cinvestav.mx
} 
where $^{*}\left(e^{I} \wedge e^{J}\right)=\frac{1}{2} \varepsilon^{I J} K L e^{K} \wedge e^{L}$ and the constants $\alpha$ and $\beta$ satisfy

$$
\frac{a_{2}}{a_{1}}=\frac{\alpha^{2}+\sigma \beta^{2}}{4 \alpha \beta}
$$

By inserting the solution (6) into (11), we get

$$
S[e, A]=\alpha \int_{\mathscr{M}^{4}}\left[*\left(e^{I} \wedge e^{J}\right)+\frac{\beta}{\alpha} e^{I} \wedge e^{J}\right] \wedge F_{I J}[A] .
$$

Before introducing the cosmological constant, it is important to remark the logic involved in the BF-like description for gravity given in Eq. (1). First of all, once we know the meaning of $Q$ 's through Eq. (6), the insertion of (6) in (3) implies that $A^{I}{ }_{J}$ is the spin connection. Therefore, the curvature $F^{I}{ }_{J}$ must satisfy Bianchi identities with no torsion $F^{I}{ }_{J} \wedge e^{J}=0$ or, equivalently, using $F_{I J}=\frac{1}{2} F_{I J K L} e^{K} \wedge e^{L}$,

$$
F_{I J K L}+F_{I K L J}+F_{I L J K}=0 .
$$

Einstein's equations in vacuum, on the other hand, are given by ${ }^{*} F^{I}{ }_{J} \wedge e^{J}=0$ or, equivalently,

$$
{ }^{*} F_{I J K L}+{ }^{*} F_{I K L J}+{ }^{*} F_{I L J K}=0
$$

where ${ }^{*} F_{I J K L}=\frac{1}{2} \varepsilon_{I J}{ }^{M N} F_{M N K L}$ is the left dual of $F_{I J K L}$. Therefore, in vacuum, Einstein's equations (10) have the same form as the Bianchi identities (9) but with $F_{I J K L}$ replaced by its left dual * $F_{I J K L}$. The relevant point in the BF description given in (10) is that the Eqs. (9) impose additional restrictions on the field $\psi_{I J K L}$ and once these are taken into account Eqs. (10) are automatically satisfied due to the use of Eq. (2), the properties of $\psi_{I J K L}$, and Eq. (5).

Now, let us come back to the issue of the cosmological constant, the heart of this paper. The inclusion of the cosmological constant $\Lambda$ modifies the right-hand side of (10), which becomes

$$
{ }^{*} F_{I J K L}+{ }^{*} F_{I K L J}+{ }^{*} F_{I L J K}=\Lambda \varepsilon_{I J K L},
$$

while (9) remains if we assume that $F_{J}^{I}$ is still the curvature of the spin connection, i.e., that there is no torsion. In order to introduce $\Lambda$ in the CMPR action principle we have at our hands the two volume terms that can be built with the $Q$ 's: $Q_{I J} \wedge Q^{I J}$ and $Q_{I J} \wedge{ }^{*} Q^{I J}$; so it looks natural to use both of them following Ref. 17 . With this in mind, we consider the modified CMPR action principle

$$
\begin{array}{r}
S[Q, A, \psi, \mu]=\int_{\mathscr{M}^{4}}\left[Q^{I J} \wedge F_{I J}[A]-\frac{1}{2} \psi_{I J K L} Q^{I J} \wedge Q^{K L}-\mu\left(a_{1} \psi_{I J}{ }^{I J}+a_{2} \psi_{I J K L} \varepsilon^{I J K L}-\mathcal{H}\right)\right. \\
\left.+l_{1} Q_{I J} \wedge Q^{I J}+l_{2} Q_{I J} \wedge^{*} Q^{I J}\right]
\end{array}
$$

where $l_{1}, l_{2}$, and $\mathcal{H}$ are constants. As it will be apparent next, the action principle (12) describes general relativity with cosmological constant $\Lambda$; the relationship among $l_{1}, l_{2}, \mathcal{H}$, and $\Lambda$ will be also displayed.

Some of the consequences of the new action principle (12) is that the equations of motion obtained from the variation of (12) with respect to the independent fields $Q^{I J}$ and $\mu$ are, instead of Eqs. (2) and (5), now given by

$$
\begin{aligned}
& \delta Q: F_{I J}[A]-\psi_{I J K L} Q^{K L}+2 l_{1} Q_{I J}+2 l_{2}{ }^{*} Q_{I J}=0, \\
& \delta \mu: a_{1} \psi_{I J}^{I J}+a_{2} \psi_{I J K L} \varepsilon^{I J K L}-\mathcal{H}=0,
\end{aligned}
$$

while the variation of (12) with respect to $A^{I}{ }_{J}$ and $\psi_{I J K L}$ gives the same equations as before, namely Eqs. (3) and (41). Therefore, Eqs. (6) and (7) are still valid for the modified action (12), and so by plugging into (12) the expression for $Q^{I J}$ in terms of the tetrad $e^{I}$, the action principle becomes

$$
S[e, A]=\int_{\mathscr{M}^{4}}\left[\left[\alpha^{*}\left(e^{I} \wedge e^{J}\right)+\beta e^{I} \wedge e^{J}\right] \wedge F_{I J}+\mu \mathcal{H}+\left(l_{1} \alpha \beta+\frac{l_{2}}{2}\left(\alpha^{2} \sigma+\beta^{2}\right)\right) \varepsilon_{I J K L} e^{I} \wedge e^{J} \wedge e^{K} \wedge e^{L}\right] .
$$

Furthermore, by inserting (6) into (13) the curvature acquires the form

$$
F_{I J K L}=2 \alpha \psi_{I J K L}^{*}+2 \beta \psi_{I J K L}-2\left(l_{1} \alpha+l_{2} \beta\right) \varepsilon_{I J K L}-2\left(l_{1} \beta+l_{2} \alpha \sigma\right)\left(\eta_{I K} \eta_{J L}-\eta_{I L} \eta_{J K}\right),
$$


with $\psi_{I J K L}^{*}=\frac{1}{2} \varepsilon_{K L}{ }^{M N} \psi_{I J M N}$ being the right dual of $\psi_{I J K L}$. Since Eqs. (6) and (3) still hold for the action principle (12) and they imply that $A^{I}{ }_{J}$ is the spin connection, then $F_{I J K L}$ must satisfy the Bianchi identities (9), like in the case without cosmological constant. Therefore, Eqs. (9) and (16) imply the following additional restrictions,

$$
2 \alpha\left(\psi_{J K L}^{* I}+\psi_{K L J}^{* I}+\psi_{L J K}^{* I}\right)+2 \beta\left(\psi_{J K L}^{I}+\psi_{K L J}^{I}+\psi_{L J K}^{I}\right)-6\left(l_{1} \alpha+l_{2} \beta\right) \varepsilon_{J K L}^{I}=0,
$$

on the components of the Lagrange multipliers $\psi_{I J K L}$, which allow us to rewrite the curvature $F_{I J K L}$ (16) as

$$
\begin{array}{r}
F_{I J K L}=2 \alpha \psi_{I J K L}^{*}+2 \beta \psi_{I J K L}-\frac{2}{3} \alpha\left(\psi_{I J K L}^{*}+\psi_{I K L J}^{*}+\psi_{I L J K}^{*}\right)-\frac{2}{3} \beta\left(\psi_{I J K L}+\psi_{I K L J}+\psi_{I L J K}\right) \\
-2\left(l_{1} \beta+l_{2} \alpha \sigma\right)\left(\eta_{I K} \eta_{J L}-\eta_{I L} \eta_{J K}\right) .
\end{array}
$$

By plugging (18) into (9) it is clear that the Bianchi identities (9) are satisfied, as it has to be, on account of the properties of $\psi_{I J K L}$.

Up to here, the Bianchi identities have been incorporated in the formalism. The remaining thing to do is to check whether Einstein's equations with cosmological constant (11) are indeed satisfied or not. In fact, the fulfilling of (11) can be accomplished by properly relating the constants $\mathcal{H}, l_{1}$, and $l_{2}$ with $\Lambda$. This is done as follows. First of all, the contraction of (17) with $\varepsilon_{I J K L}$ implies a linear equation for the two invariants $\psi_{I J}{ }^{I J}$ and $\psi_{I J K L} \varepsilon^{I J K L}$, given by

$$
\alpha \sigma \psi_{I J}{ }^{I J}+\beta \psi_{I J}^{*}{ }^{I J}-12 \sigma\left(l_{1} \alpha+l_{2} \beta\right)=0 .
$$

Nevertheless, we also have the Eq. (14) that also relates linearly the two invariants. There is no way to avoid the fulfilling of these two equations, the two must be satisfied. By combining them and using (7) it is possible to obtain

$$
\beta \psi_{I J}{ }^{I J}+\alpha \psi^{*}{ }_{I J}^{I J}=2 \beta \frac{\mathcal{H}}{a_{1}}-12 l_{1} \beta-12 l_{2} \frac{\beta^{2}}{\alpha},
$$

which is, by construction, not independent of (14) and (19). Equation (20) is a key point that allows us to include the cosmological constant in the CMPR framework because, on the other hand, Einstein's equations (11) are automatically satisfied due to the fulfilling of the Bianchi identities [or, equivalently, due to the fact that (17) or (18) holds] with the only exception, given by

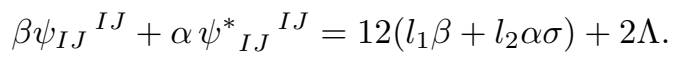

Nevertheless, by comparing (20) and (21), it is easy to see that (21) is satisfied by choosing for $\mathcal{H}$ the value

$$
\mathcal{H}=a_{1}\left[4 ! l_{2} \sigma \frac{a_{2}}{a_{1}}+12 l_{1}+\frac{\Lambda}{\beta}\right]
$$

that is to say, the choice for $\mathcal{H}$ given in Eq. (22) assures that Einstein's equations (11) are automatically satisfied.

Finally, notice that when the expressions for the $Q$ 's given in Eq. (6) and for $\mathcal{H}$ given in Eq. (22) are plugged into the action principle (12), it acquires the form in terms of the tetrad fields and the Lorentz (Euclidean) connection

$$
S[e, A]=\alpha \int_{\mathscr{M}^{4}}\left\{\left[*\left(e^{I} \wedge e^{J}\right)+\frac{\beta}{\alpha} e^{I} \wedge e^{J}\right] \wedge F_{I J}[A]-\frac{\Lambda}{12} \varepsilon_{I J K L} e^{I} \wedge e^{J} \wedge e^{K} \wedge e^{L}\right\},
$$

as expected; where it was used in the intermediate steps that $\mu a_{1}=-\frac{1}{12} Q^{I J} \wedge Q_{I J}$ and $\mu a_{2}=-\frac{\sigma}{24} Q^{I J} \wedge Q^{*}{ }_{I J}$, which come from (4).

In summary, our results are collected in the following items: (a) We have shown that by choosing the expression for $\mathcal{H}$ given in Eq. (22) and by inserting it into the action principle (12), the equations of motion that follow from this action principle are precisely Einstein's equations with cosmological constant $\Lambda$ exactly in the same way as the Plebanski action gives the equations of motion for self-dual gravity with a cosmological constant. (b) There is no need to impose by hand additional restrictions on the field variables involved to reach item (a) because the result comes out solely from the handling of the equations of motion. (c) One important feature of our construction is that we can even set $l_{1}=0=l_{2}$ from the very beginning into the action (12) (and so $\mathcal{H}=\frac{a_{1}}{\beta} \Lambda$ ) and still get the equations of motion for general relativity with cosmological constant $\Lambda$. This fact is essentially allowed by the equation given in (14), which is a modification to the equation of the pure gravity case given in Eq. (55). (d) Other particular cases are given by setting $l_{1}=0$ and $l_{2} \neq 0$ or by $l_{1} \neq 0$ and $l_{2}=0$ with the appropriate modification for $\mathcal{H}$ using (22).

To conclude, we compare our approach with the one followed in Ref. 16. The main difference between our approach and theirs is given in Eq. (14) of this paper. This fact is crucial to get precisely Einstein's equations with cosmological 
constant $\Lambda$. The authors of Ref. 16 do not use Eq. (14), rather, they use Eq. (5). This fact is apparently related to the fact that they get a cosmological constant which is a function of the Immirzi parameter. This feature is not present in our approach. Furthermore, even though they use the two volume terms constructed out of the $Q$ 's, in our approach we can get rid of them by considering the particular case in which $l_{1}=0$ and $l_{2}=0$ from the very beginning and still introduce the cosmological constant.

We think our results might be of interest for the quantum gravity community and also for the practitioners of general relativity.

Warm thanks to Riccardo Capovilla for very fruitful discussions on the subject of this paper. This work was supported in part by CONACYT, Mexico, Grant No. 56159-F.

[1] T. Thiemann, Modern Canonical Quantum General Relativity (Cambridge University Press, Cambridge, England, 2007); C. Rovelli, Quantum Gravity (Cambridge University Press, Cambridge, 2004); A. Ashtekar and J. Lewandowski, Classical Quantum Gravity 21, R53 (2004); A. Perez, in Proceedings of the International Conference on Fundamental Interactions, Domingos Martins, Brazil, (2004) arXiv:gr-qc/0409061]; A. Ashtekar, Lectures on Non-Perturbative Canonical Gravity (World Scientific, Singapore, 1991).

[2] A. Perez, Classical Quantum Gravity 20, R43 (2003); D. Oriti, Rep. Prog. Phys. 64, 1703 (2001); J.C. Baez, Lect. Notes Phys. 543, 25 (2000); Classical Quantum Gravity 15, 1827 (1998); J. Engle, R. Pereira, and C. Rovelli, Phys. Rev. Lett. 99161301 (2007); E.R. Livine and S. Speziale, Europhys. Lett. 81, 50004 (2008).

[3] V. Cuesta and M. Montesinos, Phys. Rev. D 76, 104004 (2007).

[4] V. Cuesta, M. Montesinos, M. Velázquez, and J.D. Vergara, Phys. Rev. D 78, 064046 (2008); arXiv:0809.2741.

[5] M. Montesinos and M. Velázquez, in The Planck Scale, XXV Max Born Symposium, edited by J. Kowalski-Glikman, R. Durka, and M. Szczachor, AIP Conference Proceedings 1196 (American Institute of Physics, New York, 2009) p. 201; arXiv:0812.2825.

[6] M. Montesinos and M. Velázquez, in Proceedings of the Marcel Grossmann MG12 Meeting, Paris, France, 2009.

[7] M. Montesinos, A. Perez, and M. Velázquez (work in progress).

[8] J.F. Plebański, J. Math. Phys. 18, 2511 (1977).

[9] R. Capovilla, J. Dell, T. Jacobson, and L. Mason, Classical Quantum Gravity 8, 41 (1991).

[10] M.P. Reisenberger, Classical Quantum Gravity 16, 1357 (1999).

[11] R. De Pietri and L. Freidel, Classical Quantum Gravity 16, 2187 (1999).

[12] R. Capovilla, M. Montesinos, V.A. Prieto, and E. Rojas, Classical Quantum Gravity 18, L49 (2001); arXiv:gr-qc/0102073

[13] J. F. Barbero, Phys. Rev. D 51, 5507 (1995).

[14] S. Holst, Phys. Rev. D 53, 5966 (1996).

[15] G. Immirzi, Classical Quantum Gravity 14, L177 (1997).

[16] L. Smolin and S. Speziale, Phys. Rev. D 81, 024032 (2010); arXiv:0908.3388.

[17] M. Montesinos, Classical Quantum Gravity 23, 2267 (2006); arXiv:gr-qc/0603076. 